\title{
Norms and standardizations in neuropsychology via equivalent scores: software solutions and practical guides
}

\author{
Edoardo Nicolò Aiello ${ }^{1,2}$ (1) $\cdot$ Emanuele Giovanni Depaoli ${ }^{3}$ (1)
}

Received: 13 March 2021 / Accepted: 31 May 2021 / Published online: 17 June 2021

(c) The Author(s) 2021, corrected publication 2021

\begin{abstract}
Background Norming neuropsychological tests and standardizing their raw scores are needed to draw objective clinical judgments on clients' neuropsychological profile. The Equivalent Score (ES) method is a regression-based normative/ standardization technique that relies on the non-parametric identification of the observations corresponding to the outer and inner tolerance limits (oTL; iTL) - to derive a cut-off, as well as to between-ES thresholds — to mark the passage across different levels of ability. However, identifying these observations is still a time-consuming, "manual" procedure. This work aimed at providing practitioners with a user-friendly code that helps compute TLs and ES thresholds.

Methods $\mathrm{R}$ language and RStudio environment were adopted. A function for identifying the observations corresponding to both TLs by exploiting Beta distribution features was implemented. A code for identifying the observations corresponding to ES thresholds according to a $z$-deviate-based approach is also provided.

Results An exhaustive paradigm of usage of both the aforementioned function and script has been carried out. A userfriendly, online applet is provided for the calculation of both TLs and ESs thresholds. A brief summary of the regressionbased procedure preceding the identification of TLs and ESs threshold is also given (along with an R script implementing these steps).

Discussion The present work provides with a software solution to the calculation of TLs and ES thresholds for norming/ standardizing neuropsychological tests. These software can help reduce both the subjectivity and the error rate when applying the ES method, as well as simplify and expedite its implementation.
\end{abstract}

Keywords Equivalent score $\cdot$ Neuropsychological assessment $\cdot \mathrm{R} \cdot$ Normative data $\cdot$ Tolerance limits $\cdot$ Psychometrics

\section{Introduction}

\section{Background}

When quantitatively assessing individuals' neuropsychological functioning via psychometric tests, raw scores ought to be standardized in order to: (a) draw individual-level clinical

Edoardo Nicolò Aiello

e.aiello5@campus.unimib.it

Emanuele Giovanni Depaoli

emanuelegiovanni.depaoli@phd.unipd.it

University of Milano-Bicocca, Monza, Italy

2 School of Medicine and Surgery, University of Milano-Bicocca, Monza, Italy

3 Department of Statistical Sciences, University of Padua, Padua, Italy judgments; (b) intra-/inter-individually compare performances that differ in nature and metrics [20]. For raw scores to be standardized, normative values have to be inferred first from a healthy population sample [10]. Neuropsychological data often do not meet distributional assumptions - mostly due to high inter-individual variability and ceiling/floor effects $[1,13,17]$ : non-parametric approaches are thus to be preferred when drawing norms [10]. Moreover, controls for inferential errors are needed when classifying a performance as either "normal" or defective [4].

The Equivalent Score (ES) method [5, 6, 19] standardizes regression-adjusted [14] scores into a 5-point ordinal scale that allows drawing clinical judgments: $\mathrm{ESs}=0$ and 1 meaning "defective" and "borderline," respectively; $E S=2$ meaning "low-end normal"; ESs $=3$ and 4 meaning "normal." A cut-off is identified through the outer one-sided nonparametric lower tolerance limit (oTL) - i.e., the highest ascending-order-ranked adjusted scores yielding a safety 
level $p \geq .95$ that no more than $5 \%$ of the population performs below it. The method thus inferentially addresses a performance as impaired if falling within the "worst" 5\% of the normative sample, by keeping the risk of drawing a wrong inference below 5\%. To control for inferential errors, the inner one-sided non-parametric lower TL (iTL) is also computed - i.e., the lowest observation yielding a safety level $p \geq .95$ that at most $95 \%$ of the population performs above it. Adjusted scores lower than the oTL and greater than the median are classified as ES $=0$ and 4, respectively. Between-ES conversion thresholds $(0 \rightarrow 1 ; 1 \rightarrow 2 ; 2 \rightarrow 3$; $3 \rightarrow 4$ ) are identified by subdividing into three equal segments the range of adjusted scores between the oTL and the median via a $z$-score-based approach.

\section{Aims}

The ES method is representative of regression-based approaches to norm/standardize neuropsychological tests by non-parametrically drawing cut-offs and controlling for inferential errors $[11,20]$. It is the most widely used neuropsychometric approach in Italy [2, 3]. Although whatever statistical software (e.g., R [15]) that builds in linear regressions allows identifying predictors that raw scores should be adjusted for [4], to compute TLs and ES thresholds is still currently a time-consuming, "manual" procedure. Indeed, the computerized way to identify TLs recently proposed by Capitani and Laiacona [6] is still based on a trial-and-error procedure (which exploits an online calculator provided by Casio [7]). Moreover, to the best of the authors' knowledge, no software solutions are currently available to calculate ES thresholds.
The present work thus provides practitioners with an R-based, user-friendly guide and software solution for computing TLs and ESs thresholds.

\section{Methods}

$R$ language (3.6.2) and RStudio environment were selected for writing the code since they are widely used and freely accessible $[15,16]$.

The level of R proficiency required to perform the calculations below and get the results is limited to (a) downloading R (https://cran.r-project.org) and RStudio (http:// www.rstudio.com/), (b) copying and pasting the scripts in the syntax section of RStudio and subsequently (c) running them according to the instructions provided in Figs. 1 and 2, and (d) reading the results in the output section of RStudio.

The script is mathematically adherent to the procedures described by Capitani and Laiacona [6] for identifying TLs and ES thresholds [5, 6, 19].

TLs were computed separately from ES thresholds. A function (tolLimits) yielding the observations corresponding to the outer and inner TLs along with their exact safety levels $(p)$ was implemented by adapting the iterative procedure described by Capitani and Laiacona [6] (Fig. 1). tolLimits allows calculating the observations corresponding to both TLs and respective $p$ s from a given sample size $N$.

Standard steps to identify ES thresholds from oTL and $N$ are then provided (Fig. 2):

(1) First, the cumulative density (cd1) corresponding to the oTL is computed as oTL/n;
Fig. 1 An R function to computer inner and outer tolerance limits (tolLimits). Notes. The programming lines allow to get the observations corresponding to both the outer and the inner tolerance limits along with respective safety levels. All code lines are divided in blocks to facilitate the inspection. Useful descriptions are reported in those lines introduced by a hashtag. Instructions: (1) run the first line; (2) enter the sample size $(x)$ in the last line; (3) run the last line to get the observations corresponding to tolerance limits along with respective safety levels

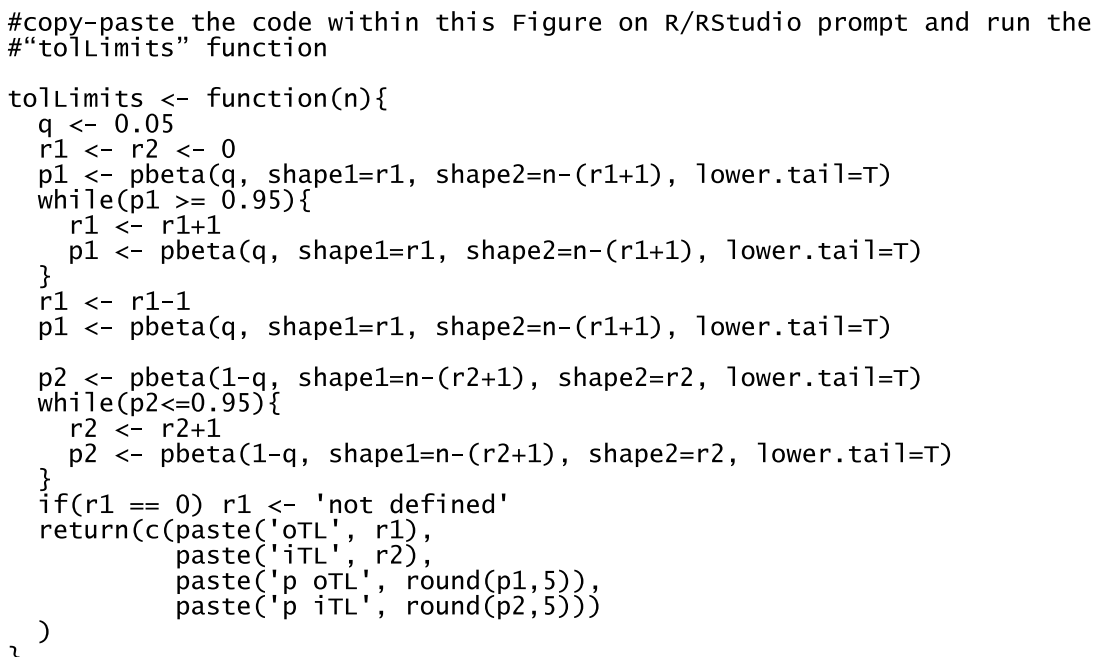

\#enter the sample size $(x)$ and run the following line to get tolerance limits. In \#the example provided below, $x$ is set to 300 . The outer and inner tolerance limits \#yie1ded wi11 thus correspond to the 9 th and 22 nd observations, respectively. The \#reader can modify these numbers to explore the effect on the results.

tolLimits $(n=300)$ 


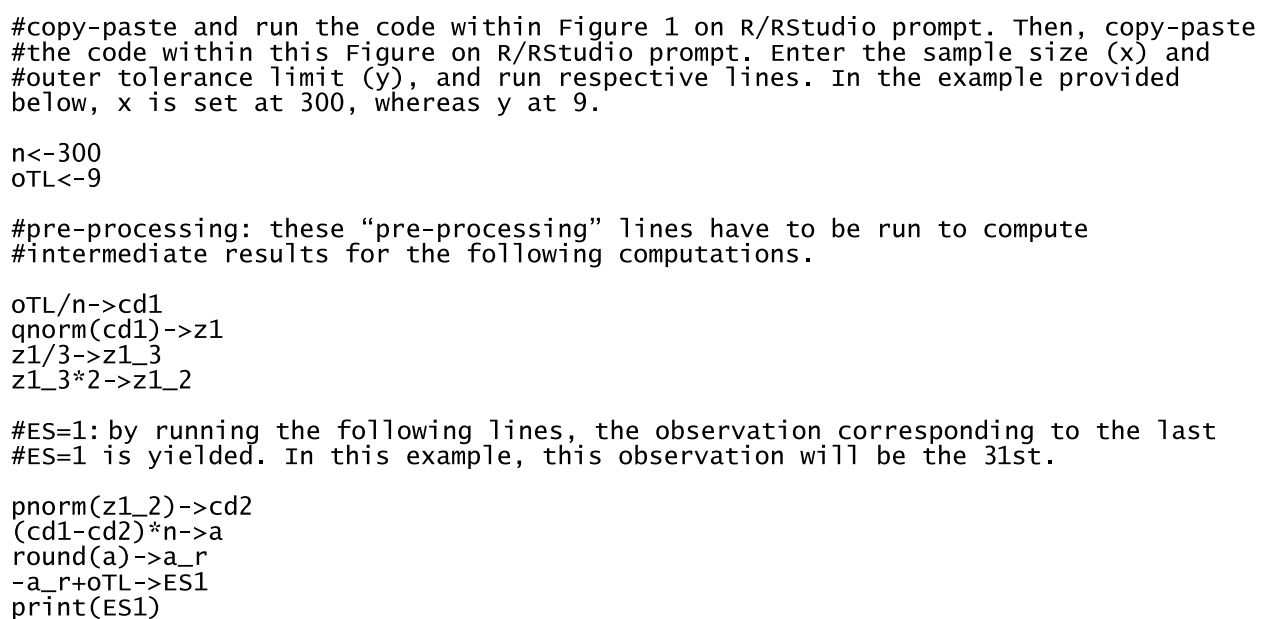

\#ctrl: these lines are allow yielding the unrounded (a) and rounded (a_r) number of \#observations falling under the $E S=1$, respectively. In this example, the rounded number wi11 be 22 .

$\operatorname{print}(a)$

print (a_r)

\#ES=2: by running the following 1ines, the observation corresponding to the 1ast

\#ES=2 is yielded. In this example, this observation will be the 79 th.

$\operatorname{pnorm}\left(z 1 \_3\right)->c d 3$

$(\mathrm{cd} 3-\mathrm{cd} 2) * \mathrm{n}->\mathrm{b}$

round (b) $\rightarrow>b_{2} r$

$\underset{b \_r+E S 1->E S 2}{\text { round }(b)->b \_r}$

print(ES2)

\#ctrl: these lines allow yielding the unrounded (b) and rounded (b_r) number of \#observations falling under the $E S=2$, respectively. In this example, the rounded \#number wi11 be 48

print (b)

print(b_r)

\#ES=3: by running the following lines, the observation corresponding to the last \#ES=3 is yielded. In this example, this observation wi11 be the 149th.

pnorm $(0)->c d 4$

$(\mathrm{cd} 4-\mathrm{cd} 3) * \mathrm{n}->\mathrm{c}$

round (c) $\rightarrow C_{-} r$

C_r $r+E S 2->E S \overline{3}$

print(ES3)

\#ctrl: these lines allow yielding the unrounded (c) and rounded (c_r) number of \#observations falling under the $E S=3$, respectively. In this example, the rounded \#number wi11 be 70 .

$\operatorname{print}(\mathrm{c})$

print (c_r)

Fig. 2 An R script to compute Equivalent Scores (ESs) thresholds. Notes. The programming lines allow to get the observations corresponding to the last Equivalent Scores $(\mathrm{ESs})=1,2$, and 3. All code lines are divided in blocks to facilitate the inspection. Useful descriptions are reported in those lines introduced by a hashtag. Instructions: (1) enter the sample size (x) and outer tolerance limit (y) and run respective lines; (2) run \#pre-processing lines; (3) run \#ES1 lines: by running print(ES1) line, the observation $\left(\mathrm{r}_{i}\right)$ corresponding to the last $\mathrm{ES}=1$ is yielded; (4) run a and a_r to get the unrounded and rounded number of $r_{i}$ s falling under the $\mathrm{ES}=1$, respectively. These "control" lines (\#ctrl) are useful to determine whether the unrounded number of $r_{i} \mathrm{~s}$ is close to the rounding threshold (.5; e.g., 25.47): this allows users to judge whether a should be rounded up or down (indeed, round() function by default rounds up numbers to the nearest integer when decimals are $\geq .5$ ). If deciding to round up a, the last $\mathrm{ES}=1$ will be equal to ES1+1; therefore, +1 will have to be added to the b_r+ES1$>$ ES2 line. Steps (3) and (4) are to be repeated on the following lines in order to get the last $\mathrm{ES}=2$ and 3. Users have to note that the applet associated with this script automatically rounds up number to the nearest integer when decimals are $\geq .5$ (according to the round() function)
(2) The $z$-deviate at cd1 is subsequently calculated via qnorm() and then divided by 3 . The three segments comprising observations that will fall within the $\mathrm{ES}=1$, 2 , and 3 are bounded by the following $z$-deviates, respectively: z1_3 (i.e., the quotient of z1/3), z1_2 (i.e., the double of $z 1 \_3$ ), and 0 ;

(3) The observation corresponding to the last $\mathrm{ES}=1$ is identified by adding the number of observations com- 
prised within the oTL to that of observations falling under the $\mathrm{ES}=1$. While the former addend is equal to the oTL itself, the latter is identified by the following: (1) computing the cumulative density at z1_2 (cd2) via pnorm(); (2) multiply by $N$ the subtraction of cd1 from cd2 (a); (3) adding the rounded a to the oTL. The same procedure is applied in order to identify the last $\mathrm{ES}=2$ and 3, with the only exception that the second addend corresponds to the number of observations falling under the $\mathrm{ES}=1$.

\section{Results}

A paradigm of usage of the scripts displayed in Figs. 1 and 2 is provided. The paradigm takes into account both categorical (e.g., sex) and continuous (e.g., years of age and education) predictors and is run by assuming that the following steps have already been implemented [4]:

(1) Testing the effect of each categorical/continuous predictor on the criterion via linear model (LM) analyses (e.g., independent samples $t$-test for sex; simple linear regression for age and education);

(2) Identifying for each predictor the transformation that best fits the shape of its relationship with the criterion - e.g., $\ln (100$-age $)$ and $\sqrt{ }($ education $)$ - i.e., the transformed predictor which yields the highest $R^{2} / \beta$;

(3) Entering predictors that independently have the largest effect into a stepwise multiple regression procedure in order to identify the best model - i.e., the one comprising only significant predictors. The $p$ value can be in this case Bonferroni-corrected $-p_{\text {adjusted }}=.05 / \mathrm{k}$ with $k$ being the number of predictors [19].

(4) Adjusting raw scores (with the exception of those corresponding to either the minimum or the maximum of the test) via the following equation yielded by the best model: $\mathrm{AS}=\mathrm{RS}+\left[-b_{1} *\left(x_{1}-M_{x 1}\right)\right]+\left[-b_{2} *\right.$ $\left.\left(x_{2}-M_{x 2}\right)\right] \ldots+\left[-b_{i} *\left(x_{i}-M_{x i}\right)\right]$; if sex is a significant predictor, the term $+\left[-b_{\text {sex }} *(\right.$ sex -.5$\left.)\right]$ can be added (with $0=$ male and $1=$ female);

(5) Ranking adjusted scores and ordering observations in ascending order by adjusted score ranks.

An R script for the implementation of the aforementioned steps is made available at https://github.com/enaiello/ES_ ENA_EGD.
Let $N=300$ be the sample size. tolLimits function will thus yield the following results: oTL $=9$ with $p=.964$ and $\mathrm{iTL}=22$ with $p=.954$. The oTL and the iTL will be thus equal to the adjusted scores corresponding to the 9th and 22nd observations, respectively. Let the range of the test $0-15$; let oTL and iTL be $=4.571$ and 5.203, respectively. Adjusted scores will be assigned an $\mathrm{ES}=0$ if $4.571 \leq$; the cut-off will thus be 4.572 . Adjusted scores between the oTL and the iTL (4.572 $\leq$ adjusted scores $\leq 5.203$ ) will lay in the so-called gray area [6] — i.e., when drawing clinical judgments based on these adjusted scores (i.e., whether a performance is impaired or not), one cannot be sure that the error risk is kept at $5 \%$.

If the oTL falls within a run of equal adjusted scores i.e., adjusted scores belonging to the same rank (tied ranks) - the highest observation below the "formal" oTL can be regarded as the "actual" oTL in order to keep the error risk $5 \%<$. Similarly, if the iTL falls within a run of tied-ranked adjusted scores, the lowest observation above the "formal" oTL can be regarded as the "actual" oTL.

The $z$-deviate corresponding to the oTL (z1) is -1.880794 (given a cumulative density cd1 of .03).z1_3 and z1_2 are then computed and $=-.6269312$ and -1.253862 , respectively. The number of observations being attributed an $\mathrm{ES}=1$ is 22 (a_r) - since the corresponding unrounded number (a) is -22.4838 . The last $\mathrm{ES}=1$ will be thus the 31 st observation - i.e., the cumulative number of observations from the lowest to this.

Similar computation will yield the number of observations falling under the $\mathrm{ES}=2$ and $3-48$ and 70 , respectively - as well as observations corresponding to the last $\mathrm{ES}=2$ and 3 the 79th and the 149th, respectively. Let the adjusted scores corresponding to the last $\mathrm{ES}=1,2$, and $3 \mathrm{be}=5.897,7.285$, and 9.771, adjusted scores being assigned an $\mathrm{ES}=1,2,3$, or 4 fall within the ranges displayed in Table 1.

If ES thresholds happened to fall within a run of equal adjusted scores, the same rule adopted with respect to the iTL can be applied (regarding the lowest observation above the "formal" ES threshold as the "actual" threshold). This expedient would make the considered ES area wider and thus allows being as conservative as possible when passing from one ES to the next (i.e., to assign a higher level of ability to an adjusted score).

tolLimits function and the script for computing ESs thresholds have been also implemented as user-friendly, online applets by means of the R package shiny [8] (retrievable at https://egdp.shinyapps.io/tolLimits/). The applet yields both TLs and the observations corresponding to the last $\mathrm{ES}=1,2$, and 3 by simply entering the sample size.
Table 1 Tolerance limits (TLs) and Equivalent Score ranges for putative adjusted scores on a test

\begin{tabular}{lllllll}
\hline Outer TL & Inner TL & \multicolumn{5}{l}{ Equivalent Scores } \\
\cline { 3 - 7 } & & 0 & 1 & 2 & 3 & 4 \\
\hline 4.571 & 5.203 & $\leq 4.571$ & $4.572-5.897$ & $5.898-7.285$ & $7.286-9.771$ & $\geq 9.772$ \\
\hline
\end{tabular}




\section{Discussion}

The present work provides practitioners in the neuropsychometric field a software solution (R scripts and user-friendly, online applets) for computing TLs and ES thresholds to norm neuropsychological tests, as well as for implementing regression-based steps that precede their calculation - this allowing to simplify and expedite the norming procedure, as well as to make it less subjected to "human" errors.

This last assertion is especially relevant when taking into account socio-demographic changes that give rise to the need of updating neuropsychological test norms [18]. Indeed, several neuropsychological tests still rely on normative data collected decades ago [2, 3, 19].

This article can be thus regarded as a "software translation" of the original ESs approach [5, 6, 19]. However, a single note should be made with regard to the median: here, it is addressed as falling within the $\mathrm{ES}=4$, according to the most recent statement by Capitani and Laiacona [6].

Moreover, practitioners should bear in mind that modifications can be adopted within the regression-based procedure preceding the calculation of TLs and ESs thresholds. First, transforming predictors can be complemented with polynomial regression analyses - which may help identifying the actual shape of the relationship between the predictor and the outcome and thus select the best transform [9]. Second, in order to select the best predictors, methods other than addressing $R^{2} / \beta$ statistics can be adopted (see Heinze et al. [12] for a comprehensive review on the topic).

In the present work, two computational issues regarding the ES method have also been approached: (a) attributing the number of observations within each ES in uncertainty scenarios (i.e., when a non-integer, close-to-rounding-threshold number is yielded); (b) defining TLs and ES threshold in the presence of tied ranks [19]. However, practitioners have to be aware of the fact that the above proposals are merely empirical, "thumb" rules. Indeed, while the former aspect is mostly subjected to each practitioner's judgment, future works are needed to provide theoretical support to the latter.

Finally, it should be highlighted that the most relevant contribution of this work to users is arguably represented by the user-friendly applet (https://egdp.shinyapps.io/tolLi mits/). This applet indeed allows to immediately get the observations corresponding to both TLs (iTL; oTL) and ES thresholds (last $\mathrm{ES}=1,2$, and 3) by simply (a) accessing the link and (b) entering the sample size.

Acknowledgements The authors would like to express their gratitude to Prof. Erminio Capitani for the insightful contributions that he provided to both the conception and the implementation of this work. The authors are also thankful to Prof. Marcello Gallucci for his insightful advice.
Funding Open access funding provided by Università degli Studi di Milano - Bicocca within the CRUI-CARE Agreement.

Code availability The complete R script described in the present work is openly retrievable on GitHub at https://github.com/enaiello/ES_ ENA_EGD. A user-friendly applet to compute TLs and ESs thresholds based on the present $\mathrm{R}$ script is accessible at https://egdp.shiny apps.io/tolLimits/.

\section{Declarations}

Ethical approval None.

Conflict of interest The authors declare no competing interests.

Open Access This article is licensed under a Creative Commons Attribution 4.0 International License, which permits use, sharing, adaptation, distribution and reproduction in any medium or format, as long as you give appropriate credit to the original author(s) and the source, provide a link to the Creative Commons licence, and indicate if changes were made. The images or other third party material in this article are included in the article's Creative Commons licence, unless indicated otherwise in a credit line to the material. If material is not included in the article's Creative Commons licence and your intended use is not permitted by statutory regulation or exceeds the permitted use, you will need to obtain permission directly from the copyright holder. To view a copy of this licence, visit http://creativecommons.org/licenses/by/4.0/.

\section{References}

1. Aiello EN, Depaoli EG, Gallucci M (2020) Usability of the negative binomial model for analyzing ceiling and highly-inter-individually-variable cognitive data. Neurol Sci 41:S273-S274

2. Bianchi A (2013) L'esame neuropsicologico dell'adulto. Giunti Psychometrics, Firenze

3. Bianchi A, Dai Prà M (2008) Twenty years after Spinnler and Tognoni: new instruments in the Italian neuropsychologist's toolbox. Neurol Sci 29:209-217

4. Capitani E (1997) Normative data and neuropsychological assessment. Common problems in clinical practice and research. Neuropsychological Rehabilitation 7:295-310

5. Capitani E, Laiacona M (1997) Composite neuropsychological batteries and demographic correction: standardization based on equivalent scores, with a review of published data. J Clin Exp Neuropsychol 19:795-809

6. Capitani E, Laiacona M (2017) Outer and inner tolerance limits: their usefulness for the construction of norms and the standardization of neuropsychological tests. Clin Neuropsychol 31:1219-1230

7. Casio (2021) Beta distribution calculator. Keisan Online Calculator. Retrieved from https://keisan.casio.com/exec/system/11805 73225. Accessed 29 Jan 2021

8. Chang W, Cheng J, Allaire JJ, Xie Y, McPherson J (2020) Shiny: web application framework for $R$. (R package version 1.5.0) [Computer software]. Available from https://CRAN.R-project. org/package=shiny. Accessed 1 Mar 2021

9. Cohen J, Cohen P, West S, Aiken L (2003) Applied multiple regression/correlation analysis for the behavioral sciences. Routledge

10. Crawford JR (2003) Psychometric foundations of neuropsychological assessment. In: Goldstein LH, McNeil J (eds) Clinical 
neuropsychology: a practical guide to assessment and management for clinicians. Wiley, Chichester, pp 121-140

11. Crawford JR, Garthwaite PH (2006) Comparing patients' predicted test scores from a regression equation with their obtained scores: a significance test and point estimate of abnormality with accompanying confidence limits. Neuropsychology 20:259-271

12. Heinze G, Wallisch C, Dunkler D (2018) Variable selection-a review and recommendations for the practicing statistician. Biom J 60:431-449

13. Malek-Ahmadi M, Mufson EJ, Perez SE, Chen K (2017) Statistical considerations for assessing cognition and neuropathology associations in preclinical Alzheimer's disease. Biostatistics \& Epidemiology 1:92-104

14. Oosterhuis HE, van der Ark LA, Sijtsma K (2016) Sample size requirements for traditional and regression-based norms. Assessment 23:191-202

15. R Core Team (2019) R: a language and environment for statistical computing. (Version 3.6.3) [Computer software]. Retrieved from https://cran.r-project.org. Accessed 29 Jan 2021

16. RStudio Team (2020) RStudio: integrated development for R. [Computer software]. Retrieved from http://www.rstudio.com/. Accessed 29 Jan 2021
17. Scherr M, Kunz A, Doll A, Mutzenbach JS, Broussalis E, Bergmann HJ, Kirschner M, Trinka E, Killer-Oberpfalzer M (2016) Ignoring floor and ceiling effects may underestimate the effect of carotid artery stenting on cognitive performance. J Neurointerv Surg 8:747-751

18. Siciliano M, Chiorri C, Battini V, Sant'Elia V, Altieri M, Trojano L, Santangelo G (2019) Regression-based normative data and equivalent scores for trail making test (TMT): an updated Italian normative study. Neurol Sci 40:469-477

19. Spinnler H, Tognoni G (1987) Standardizzazione e taratura italiana di test neuropsicologici. Ital J Neurol Sci 6:1-120

20. Willmes K (2010) The methodological and statistical foundations of neuropsychological assessment. In: Gurd J, Kischka U, Marshall J (eds) The handbook of clinical neuropsychology, 2nd edn. Oxford University Press, New York, pp 28-49

Publisher's note Springer Nature remains neutral with regard to jurisdictional claims in published maps and institutional affiliations. 